\title{
Stable Graphene Oxide Crosslinked Membranes for Organic Solvent Nanofiltration
}

\author{
Dinesh K. Mahalingam, Shaofei Wang, and Suzana P. Nunes*
}

King Abdullah University of Science and Technology (KAUST), Biological and Environmental Science and Engineering Division (BESE), Advanced Membranes and Porous Materials Center, 23955-6900 Thuwal, Saudi Arabia

KEYWORDS: graphene oxide, ethylenediamine, membrane, organic solvent, nanofiltration, stability

\begin{abstract}
Graphene Oxide (GO) membranes are well-known materials for water purification, owing to their unique molecular transport and sieving properties. However, one of the factors that limit their use in aqueous and organic solution for the application in the industry and water treatment plants is the tendency to swell, forming an enlarged interlayer spacing, disintegrating its structure. Here we report stable ethylenediamine-crosslinked GO membranes, which can efficiently permeate water and organic solvents and filter organic solutions containing molecular dyes. The prepared membranes with $150 \mathrm{~nm}$ thickness demonstrated high permeance for water and organic solvents and exhibited $>95 \%$ rejection for small organic dyes dissolved in methanol. A dead-end filtration device was used to estimate the performance of the membranes, confirming a pure water
\end{abstract}


permeance of $14 \mathrm{~L} \mathrm{~m}^{-2} \mathrm{~h}^{-1} \mathrm{bar}^{-1}$. The spacing between GO sheets enhances the solvent transport, while the crosslinker preserves the sheets from disrupting the structure. The combination of stability, high performance, and simplicity of fabrication is advantageous for the membrane potential application in aqueous and non-aqueous industrial separations.

\section{INTRODUCTION}

Chemical and petrochemical industries have a massive demand for liquid separations. Solvent resistant nanofiltration (NF) membranes, stable in aqueous and organic media, and able to withstand harsh conditions with superior permeability characteristics, could be highly advantageous in these separation processes. The energy consumption of conventional separation and purification processes is high and membrane technology could reduce it. ${ }^{1}$ For separating products from their residual reactants, catalysts, and byproducts, better membranes are required. A main challenge in developing membranes for organic solvent nanofiltration (OSN) is their robustness in organic solvents towards swelling and leaching. There is a lack of multipurpose, molecular selective membranes in this field. Synthesizing stable membranes with ideal nanostructure combined with molecular sieving abilities is crucial for industrial applications. Part of the advanced polymeric nanofiltration membranes offer excellent selectivity and reasonable scalability of the preparation process, but they often struggle with weak chemical resistance, when used in non-aqueous solvents, and membrane fouling, when used for water treatment. ${ }^{2}$ Furthermore, they might suffer from flux decline due to aging or compaction. On the other hand, inorganic ceramic membranes mostly possess excellent thermal and chemical stability, they do not compact under pressure, do not swell in organic solvents, but the membrane manufacture is costly and avoiding brittleness is also a challenge. ${ }^{3}$ Since more than a decade ago, $1 \mathrm{D}$ carbon nanotubes have been under consideration for membrane applications, with the expectation of permeating 
water extremely fast with nanosized transport channel. But vertically aligning the tubes is a complex, expensive and tedious process, which is far away from a practical application. ${ }^{4}$ As an alternative, free standing hard and thin diamond-like carbon nanosheets were reported as membranes to permeate organic solvents. ${ }^{3}$

Besides 1D carbon nanotubes, 2D graphene or graphene oxide (GO) based membranes are expected to promote high water fluxes with selectivity in the nanofiltration range. ${ }^{5-10}$ The competition with well-stablished thin-film polyamide membranes is difficult after decades of cost, scalability, and performance optimization. However, their application in non-aqueous medium might be more advantageous, since the graphene layer can add chemical and mechanical stability and NF selectivity, still keeping a high flux. In this way, the membranes can perform under harsher conditions with high performance. GO membranes decorated by oxygen groups offer novel and tunable properties with its unique two dimensional and mono atomic thick structure.

Pristine GO sheets are held together by the combination of van der Waals forces and hydrogen bonding, with an interlayer spacing ranging between 0.6 to $1.2 \mathrm{~nm}$, which can transport molecules, ions, gases, and fluids. ${ }^{11-12}$ However, the swelling of GO sheets in aqueous solution enlarges the interlayer $d$-spacing, leading to a significant expansion of the nanochannels. ${ }^{13}$ As a result, a permeation increase in applications such as gas separation ${ }^{14}$, pervaporation ${ }^{15-16}$ and ultra $^{17}$ and nanofiltration is frequently observed ${ }^{18}$. The $d$-spacing of pure GO laminates is estimated as $\sim 0.8$ $\mathrm{nm}$, whereas the reduced GO laminates have a value of $\sim 0.35 \mathrm{~nm} .{ }^{11,19}$

Ironically, GO membranes suffer from poor structural stability in aqueous environments and are unstable over a period of time. Membranes become negatively charged on hydration and eventually disintegrate due to electrostatic repulsion. ${ }^{20}$ The reaction of GO with water results in C-C bond cleavage forming vinylogous carboxylic acids. ${ }^{21}$ This makes pristine GO membranes 
useless for water-based applications. Out of many possible ways, crosslinking GO with other molecules can be an effective strategy to ensure the GO membranes integrity in water and nonaqueous solvents. ${ }^{22-26}$ For instance, crosslinking GO films with multivalent cationic metal contaminants during the GO synthesis renders significant water stability and higher stiffness. Crosslinking GO sheets with amine has been reported to increase stability, leading to highly charged GO framework membranes effective for heavy metal removal via nanofiltration. ${ }^{27}$ In another recent experiment, stable reduced GO membranes were fabricated using theanine amino acid and tannic acid, as reducing agent and crosslinker. ${ }^{28}$ Other crosslinkers based on diisocyanate, aliphatic terminal diamines, crosslinked polyimides, triethylenetetramine, cation intercalation, urea, thiourea, peptides, and functionalization with zwitterionic and tannic acid have been used to modify GO, mainly for water related applications. ${ }^{24,26,29-41}$ Our group recently tailored the structure of nanochannels by mimicking the hourglass shape of biological molecules introducing silicon-dioxide nanoparticles as spacers between layers. ${ }^{42}$ The structure combined high-solvent permeance with high selectivity.

The most significant difficulty with graphene membranes is how to keep a small channel size while maintaining the structural integrity at low cost. Diamines are found to be one of the best crosslinkers as it can covalently bind to graphene oxide, creating stable GO membranes. GO membranes were earlier reported with various $d$-spacing using different diamine monomers. ${ }^{36,43-}$ ${ }^{45} \mathrm{GO}$ membranes crosslinked with diamines were not explored to our knowledge for solventresistant applications. Herein, we report stable GO membranes crosslinked with ethylenediamine (EDA). We opt for short diamine crosslinkers, rather than the long-chain ones, since they may occupy less interlayer channel spaces to give a high solvent permeance. Moreover, compared with most of the GO membranes, which use flakes in the size of a few micrometer or even nanometer, 
we designed large-sized GO in the size of tens of micrometers, aiming to achieve better interlocked structure to meet the high rejection requirements for OSN applications.

\section{MATERIALS AND METHODS}

Graphite flakes, sulfuric acid, and 3-aminopropyltriethoxysilane were purchased from SigmaAldrich; nitric acid and hydrogen peroxide $\left(\mathrm{H}_{2} \mathrm{O}_{2}\right)$ were supplied by VWR Chemicals; potassium permanganate $\left(\mathrm{KMnO}_{4}\right)$ was from Fisher Scientific. Porous nylon membranes (Pore size $=0.22$ $\mu \mathrm{m}$ and Diameter $=47 \mathrm{~mm}$ ) were provided by GVS Filtration Inc.

Synthesis of GO. The synthesis of GO was adapted from previously reported methods. ${ }^{46-47}$ In a typical process, dry graphite flakes and concentrated sulfuric acid were mixed in a reaction vessel for 3 hours. Afterward concentrated nitric acid was added slowly and stirred for further 24 hours. The resultant graphite intercalated product mixture was thoroughly washed with water, centrifuged, and dried at $60{ }^{\circ} \mathrm{C}$. The expansion of graphite was carried out at $1050{ }^{\circ} \mathrm{C}$ for 15 seconds. During the expansion procedure, about $200 \mathrm{~mL}$ of sulfuric acid was added to and stirred well followed by addition of $\mathrm{KMnO}_{4}$. The reaction vessel was the transferred to an ice bath and stirred for further 24 hours until complete oxidation was achieved. After an incubation time of 24 hours, $200 \mathrm{~mL}$ of deionized water and $50 \mathrm{~mL}$ of $\mathrm{H}_{2} \mathrm{O}_{2}$ were added to the vessel, which resulted in a change in solution color to yellowish-brown. Later the mixture is washed with 9:1 ratio of water: $\mathrm{HCl}(\mathrm{V} / \mathrm{V})$ followed by washing with deionized water until the $\mathrm{pH}$ of the mixture changed to 5. Finally, the GO suspension was shed and diluted by gentle shake.

Synthesis of diamine-GO suspension. The synthesis process was based on two previously reported methods. ${ }^{43,48}$ In a typical procedure, the exfoliated GO suspension was further diluted to reach GO concentration of $400 \mathrm{ppm}$. Ethylenediamine (EDA) was added to the GO suspension to 
prepare a $0.1 \mathrm{M}$ solution concerning the monomer. This mixture was heated at $75^{\circ} \mathrm{C}$ for 6 hours in a sealed glass vessel followed by cooling down.

Fabrication of EDA-GO membranes. EDA-GO membranes were fabricated by a vacuum filtration method, as depicted in Figure S1. The stock was diluted to form a $0.01 \mathrm{mg} / \mathrm{mL}$ EDA-GO dispersion, by adding Milli-Q water. $20 \mathrm{~mL}$ of the $0.01 \mathrm{mg} / \mathrm{mL}$ dispersion was typically filtered on a porous nylon support membrane. The obtained membranes were further heat-treated in an oven at $80^{\circ} \mathrm{C}$. To remove the unwanted diamine monomers, the resulting membranes were soaked in methanol for $12 \mathrm{~h}$. Additionally, the membranes were heat-treated in a vacuum oven at $100{ }^{\circ} \mathrm{C}$ for over $24 \mathrm{~h}$. It is sufficient for the ring opening reaction to occur at this temperature to allow unreacted epoxides to react with GO sheets. ${ }^{43}$ Additionally, high temperature can assist in partial reduction, creating the necessary pressure within stacked layers. ${ }^{49}$ The membranes obtained here were then characterized.

\section{Morphological and spectroscopic characterization of GO and EDA-GO membranes.} Scanning electron microscopy (SEM) (FEI Nova Nano microscope, equipped with energydispersive X-ray spectroscopy (EDS)) was used to image the surface and cross-sectional morphologies of GO sheets and EDA-GO membranes. The thickness of the GO sheets, as well as EDA-GO membranes, were imaged by atomic force microscopy (AFM, Bruker Dimension Icon SPM microscope). For the thickness measurement of EDA-GO membranes, the fabrication step was carried out on anodic aluminum oxide (AAO) membrane $(0.2 \mu \mathrm{m}$ pore size, $47 \mathrm{~mm}$ diameter, supplied by Whatman).

X-ray diffraction $(\mathrm{XRD})$ studies were carried out using a powder XRD system (Bruker D8 advance) with $\mathrm{Cu} \mathrm{K} \alpha(\lambda=0.154 \mathrm{~nm})$, operating at $40 \mathrm{keV}$ with a current of $20 \mathrm{~mA}$. Attenuated Total Reflectance (ATR) Fourier Transform Infrared Spectroscopy (FTIR) (FTIR-iS10) in a 
wavenumber range of 500-4000 $\mathrm{cm}^{-1}$ at an average of $32 \mathrm{scans}$ with $4 \mathrm{~cm}^{-1}$ resolution was used to do chemical analysis. The absorption spectra of GO and EDA-GO dispersions were analyzed using UV-vis spectroscopy (UV-vis) on a Cary 100 equipment. The surface elemental compositions were determined using X-ray photoelectron spectroscopy (XPS, Amicus equipment). The birefringence study of the GO and EDA/water dispersion was examined by polarized optical microscopy (Olympus BX61 Materials Microscope).

Membrane nanofiltration performance. The membrane performance investigated using a deadend stainless-steel cell. All the experiments were carried out at room temperature and with a pressure difference of 1 bar. The concentration of dyes after and before filtration was measured using a NanoDrop UV-Vis Spectrophotometer. Three measurements were performed, and the average is reported. The pure solvent permeance is calculated from the following equation:

$$
F=\frac{\Delta w}{\rho A \Delta p \Delta t}
$$

where $\Delta \mathrm{w}$ is the increase in weight of permeate during filtration at time $\Delta \mathrm{t}, \mathrm{A}$ is the separation area of the membrane, $\rho$ is the density of solvent and $\Delta p$ is the applied pressure.

The dye rejection was calculated using equation (2):

$$
R=1-\frac{C_{p}}{C_{f}} \times 100 \%
$$

where, $\mathrm{C}_{\mathrm{p}}$ and $\mathrm{C}_{\mathrm{f}}$ are the concentration of permeate and feed solutions, respectively.

\section{RESULTS AND DISCUSSION}

Monolayer GO sheets with lateral size larger than tens of micrometers were obtained, as shown in Figure 1a. Large GO sheets stacked to form membranes have the advantage of better blocking leaky paths due to boundaries and weak mechanical properties. ${ }^{50}$ Large GO sheets are expected to stack better and align when deposited on a porous support. ${ }^{51} \mathrm{~A}$ thickness of ca. $1 \mathrm{~nm}$ was confirmed 
by atomic force microscopy (AFM) for the obtained monolayer GO sheets, as shown in Figure 1b. Herein, an EDA-GO suspension was filtered using nylon microfiltration membranes via vacuum filtration, and the thickness was controlled by optimizing the volumes of the EDA-GO suspension. A thin laminate of EDA-GO was obtained as a result of the vacuum filtration. AFM height profiles confirming the thickness of $c a .150 \mathrm{~nm}$ for freestanding EDA-GO membrane is shown in Figure 1c with their corresponding thickness measurement area (1c inset). The surface morphology of the porous nylon support and the EDA-GO layer are shown in Figure 1d. The crosssectional image of EDA-GO membrane displaying laminar structure with thickness around 150 $\mathrm{nm}$ is further confirmed in Figure 1e with detailed high magnification in Figure 1f. 

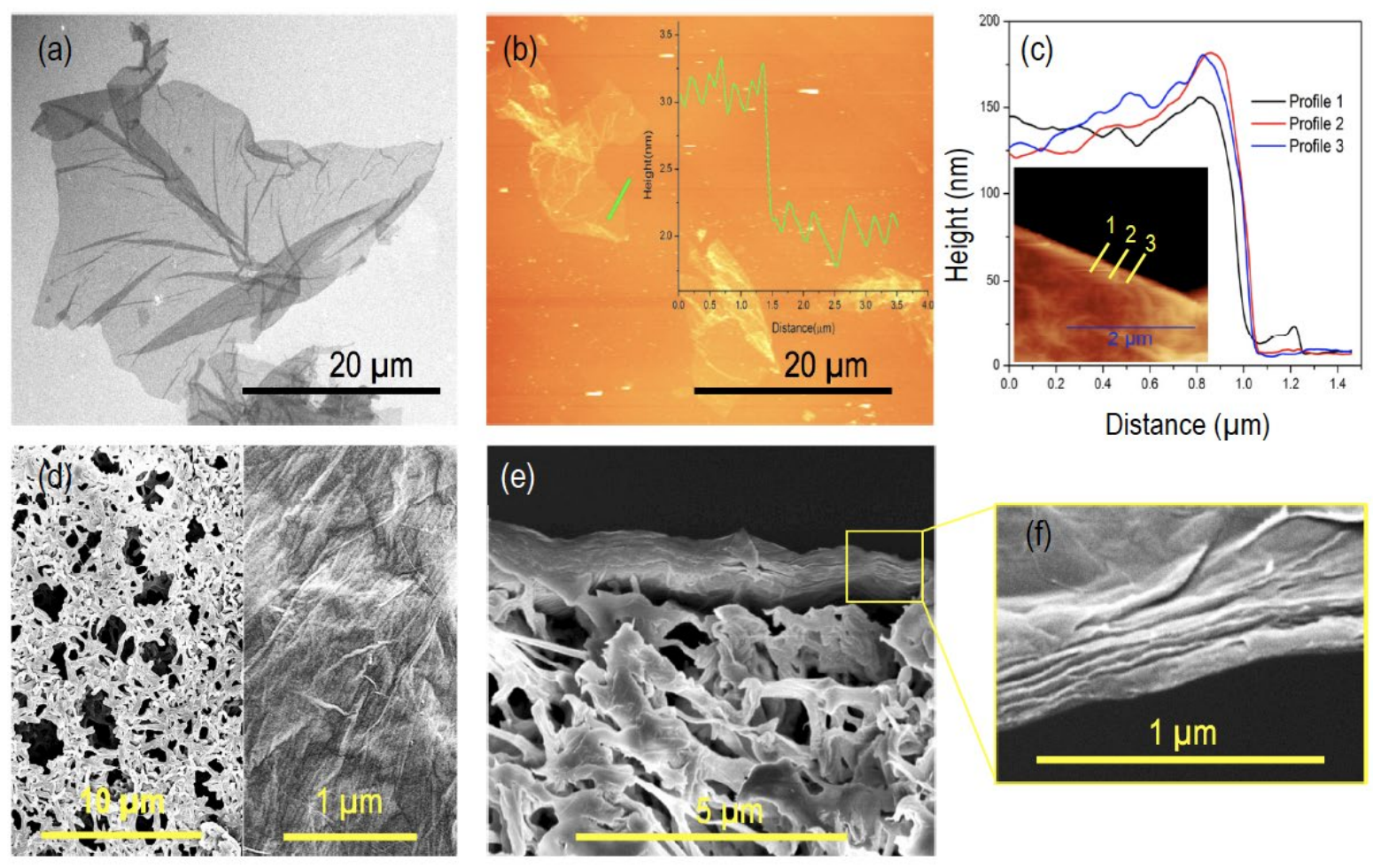

Figure 1. (a) SEM image of a free-standing GO nanosheet. (b) AFM image of GO sheets and corresponding thickness measurement. (c) Thickness measurement of the EDA-GO coating layer on the nylon support, measured by AFM (inset: AFM image corresponding to the measurement area). (d) The surface morphologies of the porous nylon support (left) and of the $150 \mathrm{~nm}$ thick EDA-GO layer (right). (e) Cross-sectional SEM image of the EDA-GO/nylon membrane, and (f) cross-sectional detail of the EDA-GO layer at higher magnification.

The interlayer spacing of GO sheets has a dominant effect on their performance. Vacuumfiltered GO and EDA-GO membranes are shown in Figure S2. Results from XRD spectra showed that the interlayer spacing increased when GO sheets were crosslinked with EDA, as shown in Figure 2a. The as synthesized GO membrane has a peak at $9.98^{\circ}$ with the corresponding $d$-spacing 
of $0.88 \mathrm{~nm}$. On the other hand, the crosslinker increased the $d$-spacing value to $1.02 \mathrm{~nm}$, by shifting the $2 \theta$ value to $8.68^{\circ}$. The molecular transport through the GO membrane is determined by the $d$ spacing between neighboring GO sheets and in-plain voids of the GO sheets. ${ }^{52}$ The chemistry between EDA crosslinked GO, and unmodified membranes can be explained by the ATR-FTIR spectra, as shown in Figure $2 \mathrm{~b}$. The stretching vibration of $\mathrm{O}-\mathrm{H}$, carboxyl $\mathrm{C}=\mathrm{O}$, aromatic $\mathrm{C}=\mathrm{C}$, epoxy C-O, and alkoxy C-O are observed at wavenumbers 3100-3400 $\mathrm{cm}^{-1}, 1720-1740 \mathrm{~cm}^{-1}, 1610$ $\mathrm{cm}^{-1}, \sim 1230 \mathrm{~cm}^{-1}, \sim 1040 \mathrm{~cm}^{-1}$, respectively. A new absorption peak at $1554 \mathrm{~cm}^{-1}$ was observed, which corresponds to amine $\mathrm{N}-\mathrm{H}$. The diamine monomer can be assumed to react with $\mathrm{GO}$ in three possible ways; (1) hydrogen bonding between oxygen groups of GO and amine molecules; (2) ionic bonding between weekly acidic sites of GO and protonating amine; (3) covalent bonding from condensation and nucleophilic substitutions reactions between amine and oxygen containing GO. ${ }^{43}$ Thus the FTIR spectra confirms the generation of new N-H covalent bond by a condensation reaction between the amine groups and the oxygen groups of GO sheets and a nucleophilic substitution reactions between amine and epoxy of GO sheets. ${ }^{53}$ GO framework enhances the stability of the membranes, preserving the sheets from disintegration in an aqueous environment.

The elemental composition of the chemical bonds for GO and EDA-GO membranes was analyzed by XPS, as shown in Figure 2c. The spectra confirmed that the N1s peak was absent in GO membranes, and only the C1s and O1s peaks are present. However, the peak for N1s in EDAGO membranes was observed, indicating that the diamine monomers reacted with the oxygen functional groups in GO. The nitrogen content in the EDA-GO membrane was $12 \%$, as shown in Table 1. Individual XPS peaks for C1s, O1s, and N1s were obtained for the membranes, as shown in Figure S3. Additionally, the oxygen content in EDA-GO membranes substantially decreased from 27 to $19 \%$ compared with the GO membranes. This occurred as a result of the crosslinking 
reaction between the oxygen-containing functional groups, and this was consistent with the obtained FTIR spectra. Hydroxyl groups were partially consumed in the reaction between the oxygen-containing groups of GO and the amine molecules. The incorporation of aminocrosslinking segments led to the nitrogen peak in XPS. The elemental mapping showed a homogeneous distribution of elements such as carbon, oxygen on the GO and EDA-GO membranes (Figure S4 and S5) and additionally a more scattered nitrogen distribution on the EDAGO membranes (Figure S5), which reflects the incorporation of nitrogen-terminated crosslinking segments. The ultraviolet-visible (UV-vis) spectra reported in Figure $2 \mathrm{~d}$ indicated two characteristic peaks, representing $\pi-\pi^{*}$ (conjugation) and $\mathrm{n}-\pi^{*}$ transitions for the GO dispersion. After the reaction, the spectrum for the EDA-GO dispersion did not have a shoulder around 300 nm. Large GO flakes leading to a discotic nematic phase of GO can be fabricated into membranes, leading to highly ordered, continuous, thin top layers as previously reported. ${ }^{47,54}$ The nematic GO dispersion taken as a precursor in this work lost its liquid crystalline property as a consequence of the EDA crosslinking with inter- and intralayers of oxygenated GO. This indicates that the EDA may perpendicularly crosslink two parallel GO sheets or two adjacent sheets. ${ }^{27}$ As a result, birefringence was observed for GO dispersion and not for EDA-GO dispersion, as shown in Figure $2 \mathrm{e}$ and $2 \mathrm{f}$, respectively. 

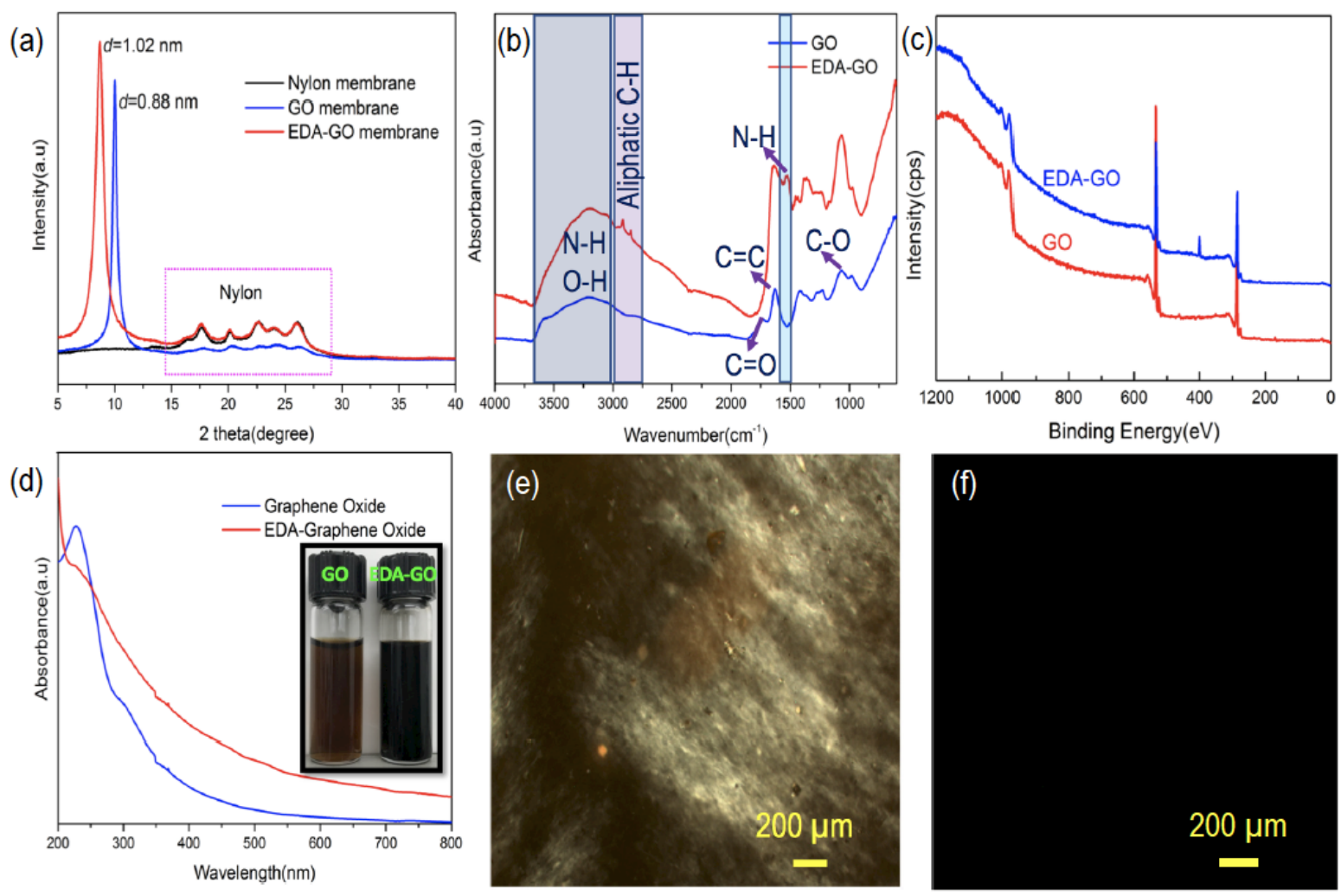

Figure 2. (a) XRD patterns of the membranes on nylon support, showing the various interlayer spacings. (b) ATR-FTIR spectra of GO and EDA-GO membranes. (c) XPS spectra of GO and EDA-GO membranes, revealing the presence of nitrogen after crosslinking. (d) UV-Vis absorption spectra for dispersion containing GO sheets and diamine monomers in EDA-GO. Inset is a photograph of the GO and EDA-GO dispersions. (e) and (f) Polarized optical microscopy images of GO and EDA-GO dispersion, indicating the presence and absence of liquid crystallinity, respectively. 
Table 1. XPS elemental composition of GO and EDA-GO membranes before and after crosslinking.

\begin{tabular}{lllll}
\hline Sample & $\mathbf{C} \%$ & $\mathbf{O} \%$ & $\mathbf{N} \%$ & $\mathbf{O} / \mathbf{C}$ \\
\hline GO & 72.73 & 27.27 & - & 0.37 \\
EDA-GO & 68.26 & 19.36 & 12.38 & 0.28 \\
\hline
\end{tabular}

\section{Nanofiltration in Non-Aqueous Solvents}

When filtered through the GO membranes, water, and other small molecules go through nanochannels and holes in the graphene sheets and reach the pores of the support membranes. The separation performance in non-aqueous solvents can be less predictable due to differences of wettability, polarity, and swelling. ${ }^{52}$ Unlike graphene, for which the slip flow theory is the basis for fast water transport, the interactions involving the $\mathrm{GO}$ interfaces is different due to the presence of large proportion of oxygen functional groups that leads to high friction between water molecules and flow channels. The permeance of non-aqueous solvents depends on their viscosity, molecular weight, as well as the interaction between the solvent molecules and membranes. ${ }^{55}$ The latter is evident in the case of polymeric membranes. In the case of GO, it will depend on the degree of functionalization.

EDA-GO membranes exhibited pure water permeance of $14 \mathrm{~L} \mathrm{~m}^{-2} \mathrm{~h}^{-1} \mathrm{bar}^{-1}$, as shown in Figure 3a, while the permeance for pristine GO membranes was $9 \mathrm{~L} \mathrm{~m}^{-2} \mathrm{~h}^{-1}$ bar $^{-1}$. Changes in the interlayer spacing between adjacent GO sheets $(0.8 \mathrm{~nm}$ for dry GO film) can be explained by the fact that the solution entering the GO layers could destroy part of the $\pi-\pi$ interactions and hydrogen bonding, thus stretching the $d$-spacing. Consequently, the GO membrane can lose its structure in aqueous environments as a result of the membrane swelling, becoming useless for water related applications. However, for the EDA crosslinked GO membrane, the formed N-H covalent bonds 
can resist the $d$-spacing stretching. ${ }^{43}$ The highest permeance was observed for the solvents with the lowest viscosity. The crosslinked EDA provides stability to GO membranes, making it stable in an aqueous and non-aqueous environment. The introduction of the crosslinker increased the distance between the sheets, as measured in XRD. The liquid permeance with some commonly used solvents was evaluated as a function of the inverse of the viscosity for $150 \mathrm{~nm}$ thin EDA-GO membranes, as shown in Figure 3b. Due to the increase in the d-spacing value, the EDA-GO membrane exhibited high acetone permeance of $30 \mathrm{~L} \mathrm{~m}^{-2} \mathrm{~h}^{-1} \mathrm{bar}^{-1}$. The same membrane exhibited NMP solvent permeance of $5 \mathrm{~L} \mathrm{~m}^{-2} \mathrm{~h}^{-1} \mathrm{bar}^{-1}$. The dependence is not far from linearity and infers that the viscosity of the solvents is a significant factor determining the permeance. The membranes exhibited stability and were highly permeable to the tested solvents. It is worth noting that besides the viscosity, the polarity contributes to the solvent permeance. Molecular selectivity is also essential in nanofiltration. The ultimate nanofiltration performance of EDA-GO membranes was evaluated by rejecting commonly used dye molecules dissolved in the methanol solvent, as presented in Figure $3 \mathrm{c}$ and S6. The feed and permeate solutions were analyzed using UV-Vis spectroscopy. We observed $>90 \%$ rejection for molecules higher than $826 \mathrm{~g} / \mathrm{mol}$. This shows that EDA-GO membranes were not only much more stable than the unmodified GO analogs but also highly selective. This demonstrates the importance of crosslinking. The stability of EDA-GO membranes can be attributed to the interlayer crosslinking of diamine monomers between the nanochannels, preventing the GO sheets from swelling and shrinking and making them appropriate for nanofiltration in aqueous and non-aqueous media. 


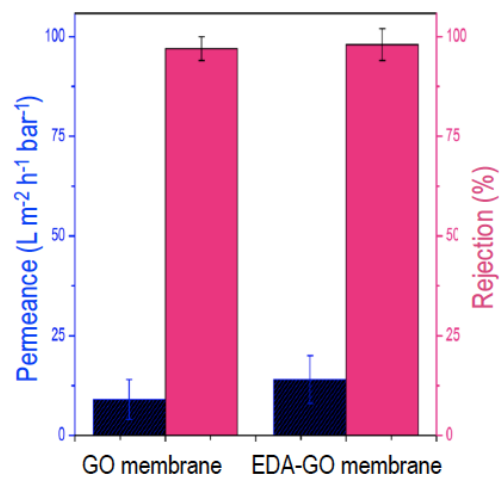

(a)

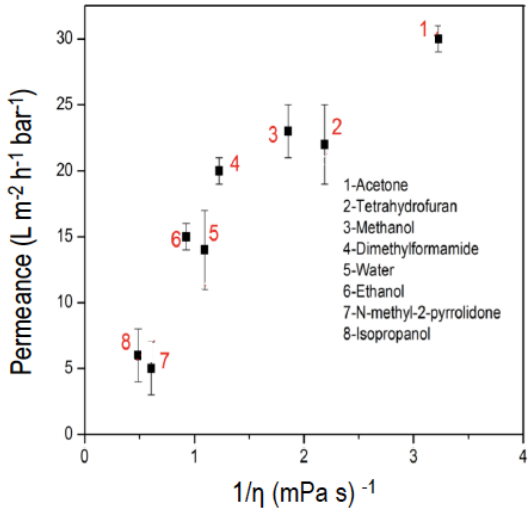

(b)

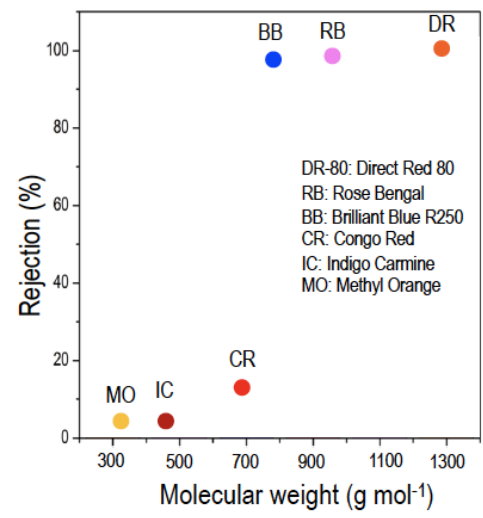

(c)

Figure 3. (a) Water permeance and Rose Bengal rejection of GO and EDA-GO membranes. (b) The permeance of pure solvents as a function of their inverse viscosity for EDA-GO membrane. (c) Dyes rejection (in methanol) as a function of the dye molecular weight for EDA-GO membrane. Dyes: Methyl Orange (MO) (MW=327 g/mol), Indigo Carmine (IC) (MW=466 g/mol), Congo Red (CR) (MW=697 g/mol), Brilliant Blue R250 (BB) (MW=826 g/mol), Rose Bengal (RB) $(\mathrm{MW}=974 \mathrm{~g} / \mathrm{mol})$, Direct Red 80 (DR-80) (MW=1373 g/mol).

\section{Potential applications of EDA-GO membranes}

A stability test was performed with water, by measuring the permeance of a EDA-GO membrane for 14 hours, as shown in Figure 4a. The membrane exhibited a steady and a stable permeance over time, which can be attributed to the effective crosslinking. Pristine GO membranes were not stable, when operated under similar conditions and this can be visually observed (inset in Figure 4a). Additionally, a model neutral solute, Vitamin $B_{12}(V B 12)(M W=1355 \mathrm{~g} / \mathrm{mol})$, was chosen to demonstrate the efficiency of the membranes for separating active pharmaceutical ingredients in organic solvents. EDA-GO membranes exhibited a $>99 \%$ rejection for VB12 in methanol with a permeance of $6.5 \mathrm{~L} \mathrm{~m}^{-2} \mathrm{~h}^{-1} \mathrm{bar}^{-1}$, as shown in Figure 4b. The crosslinked membrane can effectively reject VB12 molecules. The potential molecular sieving behavior was tested by separating a mixed 
methanol solution of Evans Blue (EB) and methyl orange (MO), both containing negative charged molecules with different molecular sizes. The rejection performance of EDA-GO membranes for EB and MO mixed solution was $>99.9 \%$ and $<10 \%$, respectively, as shown in Figure 4c. The crosslinked membrane blocked EB molecules, while selectively allowing MO molecules to permeate through the membranes. The membranes had a selective rejection based on the molecular weight. The permeances of the pristine GO membranes were low in organic solvent such as NMP $\left(<2 \mathrm{~L} \mathrm{~m}^{-2} \mathrm{~h}^{-1}\right.$ bar $\left.^{-1}\right)$ and acetone $\left(11 \mathrm{~L} \mathrm{~m}^{-2} \mathrm{~h}^{-1}\right.$ bar $\left.^{-1}\right)$, compared with higher values for EDA-GO (Fig. 3b). The increase of the interlayer $d$-spacing by crosslinking added stability to the membrane, which is crucial for operating the membranes in real time practical applications.

\section{Transport and Separation Mechanism of EDA-GO membranes}

Here the EDA crosslinker acts as a spacer, allowing aqueous and non-aqueous solvents to intercalate through GO nanochannels and transporting solvents. In addition to the GO interlayer nanocapillaries, intrinsically generated paths between sheets with a diameter in the range of nanometers also contribute for the molecular transport. ${ }^{10,56}$ The solvent flow through laminated GO sheets can be explained from Hagen Poiseuille's equation as shown in Equation 3.

$$
\text { Flux }=\frac{h^{4} \times \Delta P}{12 L^{2} \times \eta \times \Delta x}
$$

where $h$ is the $d$-spacing between adjacent GO layers, $L$ is the average lateral length of GO sheets, $\Delta P$ is the applied pressure, $\eta$ is the viscosity of solution, $\Delta x$ is the thickness of GO membranes. In the case of non-aqueous solvents, increasing the viscosity decreases the permeance, following the Hagen Poiseuille equation. 


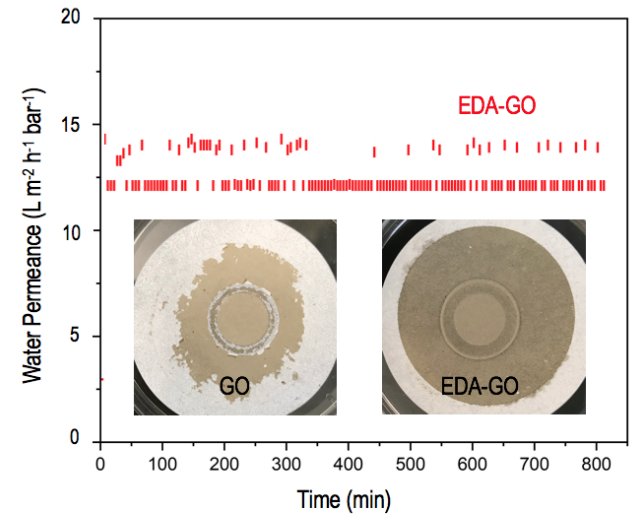

(a)

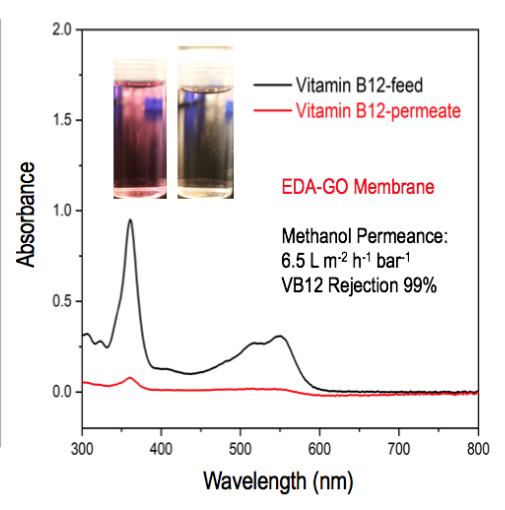

(b)

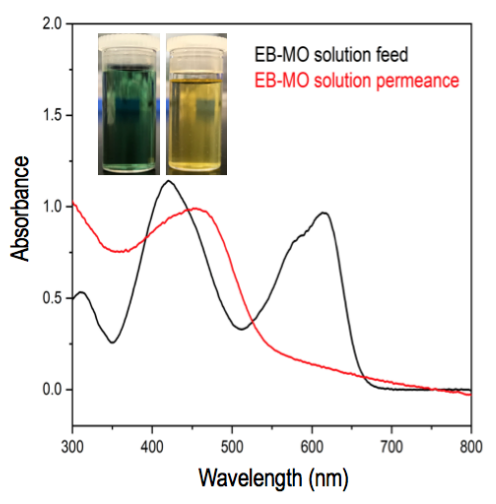

(c)

Figure 4. (a) Permeance measurement as a function of time for a EDA-GO membrane; the inset shows the pristine GO and EDA-GO membrane after measuring the water permeance. (b) Rejection of VB12 (MW=1355 g/mol): UV-vis absorbance curves of VB12 solutions in methanol used as feed and the rejected one for a EDA-GO membrane; the inset shows photographs of the feed and permeance. (c) Rejection performance for EDA-GO membrane for the filtration of a mixed dye solution of Evans Blue (MW=961 g/mol) and Methyl Orange (MW=327 g/mol) in methanol, using an EDA-GO membrane. The inset shows photographs of the feed and permeant solutions.

The separation mechanism of membranes can be attributed to: (1) size exclusion by the GO interlayer channels, (2) electrostatic interaction between negatively charged GO and incoming solute molecules, and (3) ion adsorption through binding. ${ }^{57}$ From the obtained rejection values, it is evident that size exclusion is the primary separation mechanism involved in separating solutes based on their molecular sizes. Larger solutes with high molecular weight were completely separated, while allowing solutes with low molecular weight to pass. In addition to that, most of 
the rejected dyes are negatively charged. Hence, the negatively charged hydrophilic EDA-GO membranes can electrostatically repel them. The electrostatic interaction facilitates the separation. Therefore, the selectivity of EDA-GO membranes is based both on molecular size and charge.

\section{CONCLUSION}

In conclusion, we have successfully prepared crosslinked, stable, $150 \mathrm{~nm}$ thin EDA-GO membranes by a vacuum-assisted filtration method. The membranes had an excellent performance for the retention of organic dyes dissolved in methanol with high permeance of water and nonaqueous solvents. The enlarged interlayer $d$-spacing between the GO sheets improved the permeance, while the crosslinked diamine monomer between nanosheets prevented the sheets from swelling and disintegrating. Additionally, the membranes were stable in aqueous and non-aqueous solvents, a benefit for chemical separations.

\section{ASSOCIATED CONTENT}

\section{Supporting Information}

The Supporting Information is available free of charge.

Additional experimental data: XPS spectra for elements representing C1s, O1s and N1s, digital images of membranes, elemental distribution of carbon, oxygen and nitrogen of pristine and crosslinked membranes, digital image showing rejection performance of the membranes.

\section{AUTHOR INFORMATION}

\section{Corresponding Author}


*e-mail: suzana.nunes@,kaust.edu.sa (S.P.N).

\section{ORCID}

Suzana P. Nunes: 0000-0002-3669-138X

\section{Author Contributions}

The manuscript was written through contributions of all authors. All authors have given approval to the final version of the manuscript.

\section{Notes}

The authors declare no competing financial interest.

\section{ACKNOWLEDGMENTS}

The authors thanks King Abdullah University of Science and Technology for the financial support, CRG6 grant URF/1/3441-01-01.

\section{REFERENCES}

(1) Marchetti, P.; Jimenez Solomon, M. F.; Szekely, G.; Livingston, A. G., Molecular Separation with Organic Solvent Nanofiltration: A Critical Review. Chem. Rev. 2014, 114, 10735-10806.

(2) Van der Bruggen, B.; Mänttäri, M.; Nyström, M., Drawbacks of Applying Nanofiltration and How to Avoid Them: A Review. Sep. Purif. Technol. 2008, 63, 251-263. 
(3) Karan, S.; Samitsu, S.; Peng, X.; Kurashima, K.; Ichinose, I., Ultrafast Viscous

Permeation of Organic Solvents through Diamond-Like Carbon Nanosheets. Science 2012, 335, 444-447.

(4) Yu, M.; Funke, H. H.; Falconer, J. L.; Noble, R. D., High Density, Vertically-Aligned Carbon Nanotube Membranes. Nano Lett. 2009, 9, 225-229.

(5) Wang, E. N.; Karnik, R., Water Desalination: Graphene Cleans up Water. Nat. Nanotechnol. 2012, 7, 552-4.

(6) Elimelech, M.; Phillip, W. A., The Future of Seawater Desalination: Energy, Technology, and the Environment. Science 2011, 333, 712-717.

(7) Werber, J. R.; Osuji, C. O.; Elimelech, M., Materials for Next-Generation Desalination and Water Purification Membranes. Nat. Rev. Mater. 2016, 1, 16018.

(8) Cohen-Tanugi, D.; Grossman, J. C., Water Desalination across Nanoporous Graphene. Nano Lett. 2012, 12, 3602-3608.

(9) Huang, L.; Chen, J.; Gao, T.; Zhang, M.; Li, Y.; Dai, L.; Qu, L.; Shi, G., Reduced Graphene Oxide Membranes for Ultrafast Organic Solvent Nanofiltration. Adv. Mater. 2016, 28, 8669-8674.

(10) Yang, Q.; Su, Y.; Chi, C.; Cherian, C. T.; Huang, K.; Kravets, V. G.; Wang, F. C.; Zhang, J. C.; Pratt, A.; Grigorenko, A. N.; Guinea, F.; Geim, A. K.; Nair, R. R., Ultrathin Graphene-Based Membrane with Precise Molecular Sieving and Ultrafast Solvent Permeation. Nat. Mater. 2017, 16, 1198-1202.

(11) Joshi, R. K.; Carbone, P.; Wang, F. C.; Kravets, V. G.; Su, Y.; Grigorieva, I. V.; Wu, H. A.; Geim, A. K.; Nair, R. R., Precise and Ultrafast Molecular Sieving through Graphene Oxide Membranes. Science 2014, 343, 752-754.

(12) Abraham, J.; Vasu, K. S.; Williams, C. D.; Gopinadhan, K.; Su, Y.; Cherian, C. T.; Dix, J.; Prestat, E.; Haigh, S. J.; Grigorieva, I. V.; Carbone, P.; Geim, A. K.; Nair, R. R., Tunable Sieving of Ions Using Graphene Oxide Membranes. Nat. Nanotechnol. 2017, 12, 546-550. 
(13) Hu, M.; Mi, B., Enabling Graphene Oxide Nanosheets as Water Separation Membranes. Environ. Sci. Technol. 2013, 47, 3715-3723.

(14) Li, H.; Song, Z.; Zhang, X.; Huang, Y.; Li, S.; Mao, Y.; Ploehn, H. J.; Bao, Y.; Yu, M., Ultrathin, Molecular-Sieving Graphene Oxide Membranes for Selective Hydrogen Separation. Science 2013, 342, 95-98.

(15) Liu, R.; Arabale, G.; Kim, J.; Sun, K.; Lee, Y.; Ryu, C.; Lee, C., Graphene Oxide Membrane for Liquid Phase Organic Molecular Separation. Carbon 2014, 77, 933-938.

(16) Huang, K.; Liu, G.; Lou, Y.; Dong, Z.; Shen, J.; Jin, W., A Graphene Oxide Membrane with Highly Selective Molecular Separation of Aqueous Organic Solution. Angew. Chem. Int. Ed. 2014, 126, 7049-7052.

(17) Mahalingam, D. K.; Kim, D.; Nunes, S. P., Polyethersulfone/Graphene Oxide Ultrafiltration Membranes from Solutions in Ionic Liquid. MRS Adv. 2017, 2, 2505-2511.

(18) Zhang, Y.; Chung, T. S., Graphene Oxide Membranes for Nanofiltration. Curr. Opin. Chem. Eng. 2017, 16, 9-15.

(19) Lyu, J.; Wen, X.; Kumar, U.; You, Y.; Chen, V.; Joshi, R. K., Separation and Purification Using Go and R-Go Membranes. RSC Adv. 2018, 8, 23130-23151.

(20) Yeh, C. N.; Raidongia, K.; Shao, J.; Yang, Q. H.; Huang, J., On the Origin of the Stability of Graphene Oxide Membranes in Water. Nat. Chem. 2014, 7, 166-70.

(21) Dimiev, A. M.; Alemany, L. B.; Tour, J. M., Graphene Oxide. Origin of Acidity, Its Instability in Water, and a New Dynamic Structural Model. ACS Nano 2012, 7, 576-588.

(22) Gao, T. T.; Wu, H. B.; Tao, L.; Qu, L. T.; Li, C., Enhanced Stability and Separation Efficiency of Graphene Oxide Membranes in Organic Solvent Nanofiltration. J. Mater. Chem. A 2018, 6, 19563-19569. 
(23) Li, Y.; Li, C.; Li, S.; Su, B.; Han, L.; Mandal, B., Graphene Oxide (Go)-Interlayered Thin-Film Nanocomposite (Tfn) Membranes with High Solvent Resistance for Organic Solvent Nanofiltration (Osn). J. Mater. Chem. A 2019, 7, 13315-13330.

(24) Liu, M. L.; Wang, J.; Guo, J. L.; Lu, T. D.; Cao, X. L.; Sun, S. P., Graphene Oxide/Cross-Linked Polyimide (Go/Clpi) Composite Membranes for Organic Solvent Nanofiltration. Chem. Eng. Res. Des. 2019, 146, 182-189.

(25) Rajesh, S.; Bose, A. B., Development of Graphene Oxide Framework Membranes Via the "from" and "to" Cross-Linking Approach for Ion-Selective Separations. ACS Appl. Mater. Inter. 2019, 11, 27706-27716.

(26) Liu, M.-L.; Guo, J.-L.; Japip, S.; Jia, T.-Z.; Shao, D.-D.; Zhang, S.; Li, W.-J.; Wang, J.; Cao, X.-L.; Sun, S.-P., One-Step Enhancement of Solvent Transport, Stability and Photocatalytic Properties of Graphene Oxide/Polyimide Membranes with Multifunctional Cross-Linkers. $J$. Mater. Chem. A 2019, 7, 3170-3178.

(27) Zhang, Y.; Zhang, S.; Chung, T. S., Nanometric Graphene Oxide Framework Membranes with Enhanced Heavy Metal Removal Via Nanofiltration. Environ. Sci. Technol. 2015, 49, 10235-10242.

(28) Thebo, K. H.; Qian, X.; Zhang, Q.; Chen, L.; Cheng, H. M.; Ren, W., Highly Stable Graphene-Oxide-Based Membranes with Superior Permeability. Nat. Commun. 2018, 9, 1486.

(29) Zhang, P.; Gong, J. L.; Zeng, G. M.; Deng, C. H.; Yang, H. C.; Liu, H. Y.; Huan, S. Y., Cross-Linking to Prepare Composite Graphene Oxide-Framework Membranes with High-Flux for Dyes and Heavy Metal Ions Removal. Chem. Eng. J. 2017, 322, 657-666.

(30) Qian, Y.; Zhang, X.; Liu, C.; Zhou, C.; Huang, A., Tuning Interlayer Spacing of Graphene Oxide Membranes with Enhanced Desalination Performance. Desalination 2019, 460, 56-63.

(31) Ghaffar, A.; Zhang, L.; Zhu, X.; Chen, B., Scalable Graphene Oxide Membranes with Tunable Water Channels and Stability for Ion Rejection. Environ. Sci. Nano 2019, 6, 904-915. 
(32) Zhang, Y.; Su, K.; Li, Z., Graphene Oxide Composite Membranes Cross-Linked with Urea for Enhanced Desalting Properties. J. Membr. Sci. 2018, 563, 718-725.

(33) Yang, J.; Gong, D.; Li, G.; Zeng, G.; Wang, Q.; Zhang, Y.; Liu, G.; Wu, P.; Vovk, E.; Peng, Z.; Zhou, X.; Yang, Y.; Liu, Z.; Sun, Y., Self-Assembly of Thiourea-Crosslinked Graphene Oxide Framework Membranes toward Separation of Small Molecules. Adv. Mater. 2018, 30, 1705775 .

(34) Seon, L. C.; Moon-ki, C.; Young, H. Y.; Hyunki, K.; Ki, K. M.; Jung, L. Y., Facilitated Water Transport through Graphene Oxide Membranes Functionalized with AquaporinMimicking Peptides. Adv. Mater. 2018, 30, 1705944.

(35) Qian, Y.; Zhou, C.; Huang, A., Cross-Linking Modification with Diamine Monomers to Enhance Desalination Performance of Graphene Oxide Membranes. Carbon 2018, 136, 28-37.

(36) Meng, N.; Zhao, W.; Shamsaei, E.; Wang, G.; Zeng, X. K.; Lin, X. C.; Xu, T. W.; Wang, H. T.; Zhang, X. W., A Low-Pressure Go Nanofiltration Membrane Crosslinked Via Ethylenediamine. J. Membr. Sci. 2018, 548, 363-371.

(37) Mahdavi, H.; Rahimi, A., Zwitterion Functionalized Graphene Oxide/Polyamide Thin Film Nanocomposite Membrane: Towards Improved Anti-Fouling Performance for Reverse Osmosis. Desalination 2018, 433, 94-107.

(38) Hung, W. S.; Lin, T. J.; Chiao, Y. H.; Sengupta, A.; Hsiao, Y. C.; Wickramasinghe, S. R.; Hu, C. C.; Lee, K. R.; Lai, J. Y., Graphene-Induced Tuning of the D-Spacing of Graphene Oxide Composite Nanofiltration Membranes for Frictionless Capillary Action-Induced Enhancement of Water Permeability. J. Mater. Chem. A 2018, 6, 19445-19454.

(39) Fei, F.; Cseri, L.; Szekely, G.; Blanford, C. F., Robust Covalently Cross-Linked Polybenzimidazole/Graphene Oxide Membranes for High-Flux Organic Solvent Nanofiltration. ACS Appl. Mater. Inter. 2018, 10, 16140-16147. 
(40) Ying, Y.; Liu, D.; Zhang, W.; Ma, J.; Huang, H.; Yang, Q.; Zhong, C., High-Flux Graphene Oxide Membranes Intercalated by Metal- Organic Framework with Highly Selective Separation of Aqueous Organic Solution. ACS Appl. Mater. Inter. 2017, 9, 1710-1718.

(41) Lim, M. Y.; Choi, Y. S.; Kim, J.; Kim, K.; Shin, H.; Kim, J. J.; Shin, D. M.; Lee, J. C., Cross-Linked Graphene Oxide Membrane Having High Ion Selectivity and Antibacterial Activity Prepared Using Tannic Acid-Functionalized Graphene Oxide and Polyethyleneimine. $J$. Membr. Sci. 2017, 521, 1-9.

(42) Wang, S. F.; Mahalingam, D.; Sutisna, B.; Nunes, S. P., 2D-Dual-Spacing Channel Membranes for High Performance Organic Solvent Nanofiltration. J. Mater. Chem. A 2019, 7 , 11673-11682.

(43) Hung, W.-S.; Tsou, C.-H.; De Guzman, M.; An, Q.-F.; Liu, Y.-L.; Zhang, Y.-M.; Hu, C.C.; Lee, K.-R.; Lai, J.-Y., Cross-Linking with Diamine Monomers to Prepare Composite Graphene Oxide-Framework Membranes with Varying D-Spacing. Chem. Mater. 2014, 26, 2983-2990.

(44) Xia, S.; Ni, M.; Zhu, T.; Zhao, Y.; Li, N., Ultrathin Graphene Oxide Nanosheet Membranes with Various D-Spacing Assembled Using the Pressure-Assisted Filtration Method for Removing Natural Organic Matter. Desalination 2015, 371, 78-87.

(45) Stine, R.; Ciszek, J. W.; Barlow, D. E.; Lee, W. K.; Robinson, J. T.; Sheehan, P. E., High-Density Amine-Terminated Monolayers Formed on Fluorinated CVD-Grown Graphene. Langmuir 2012, 28, 7957-61.

(46) Aboutalebi, S. H.; Gudarzi, M. M.; Zheng, Q. B.; Kim, J. K., Spontaneous Formation of Liquid Crystals in Ultralarge Graphene Oxide Dispersions. Adv. Funct. Mater. 2011, 21, 29782988.

(47) Mahalingam, D. K.; Wang, S.; Nunes, S. P., Graphene Oxide Liquid Crystal Membranes in Protic Ionic Liquid for Nanofiltration. ACS Appl. Nano Mater. 2018, 1, 4661-4670. 
Wang, Z.; Dong, Y.; Li, H.; Zhao, Z.; Wu, H. B.; Hao, C.; Liu, S.; Qiu, J.; Lou, X. W., Enhancing Lithium-Sulphur Battery Performance by Strongly Binding the Discharge Products on Amino-Functionalized Reduced Graphene Oxide. Nat. Commun. 2014, 5, 5002.

(49) Schniepp, H. C.; Li, J.-L.; McAllister, M. J.; Sai, H.; Herrera-Alonso, M.; Adamson, D. H.; Prud'homme, R. K.; Car, R.; Saville, D. A.; Aksay, I. A., Functionalized Single Graphene Sheets Derived from Splitting Graphite Oxide. J. Phys. Chem. B 2006, 110, 8535-8539.

(50) Dong, L.; Yang, J.; Chhowalla, M.; Loh, K. P., Synthesis and Reduction of Large Sized Graphene Oxide Sheets. Chem. Soc. Rev. 2017, 46, 7306-7316.

(51) Lin, X.; Shen, X.; Zheng, Q.; Yousefi, N.; Ye, L.; Mai, Y.-W.; Kim, J.-K., Fabrication of Highly-Aligned, Conductive, and Strong Graphene Papers Using Ultralarge Graphene Oxide Sheets. ACS Nano 2012, 6, 10708-10719.

(52) Akbari, A.; Meragawi, S. E.; Martin, S. T.; Corry, B.; Shamsaei, E.; Easton, C. D.; Bhattacharyya, D.; Majumder, M., Solvent Transport Behavior of Shear Aligned Graphene Oxide Membranes and Implications in Organic Solvent Nanofiltration. ACS Appl. Mater. Inter. 2018, 10, 2067-2074.

(53) Yang, Y.; Wang, J.; Zhang, J.; Liu, J.; Yang, X.; Zhao, H., Exfoliated Graphite Oxide Decorated by Pdmaema Chains and Polymer Particles. Langmuir 2009, 25, 11808-11814.

(54) Akbari, A.; Sheath, P.; Martin, S. T.; Shinde, D. B.; Shaibani, M.; Banerjee, P. C.; Tkacz, R.; Bhattacharyya, D.; Majumder, M., Large-Area Graphene-Based Nanofiltration Membranes by Shear Alignment of Discotic Nematic Liquid Crystals of Graphene Oxide. Nat. Commun. 2016, 7, 10891.

(55) Karan, S.; Jiang, Z.; Livingston, A. G., Sub-10 Nm Polyamide Nanofilms with Ultrafast Solvent Transport for Molecular Separation. Science 2015, 348, 1347-1351.

(56) An, D.; Yang, L.; Wang, T. J.; Liu, B. Y., Separation Performance of Graphene Oxide Membrane in Aqueous Solution. Ind. Eng. Chem. Res. 2016, 55, 4803-4810. 
(57) Perreault, F.; Fonseca de Faria, A.; Elimelech, M., Environmental Applications of Graphene-Based Nanomaterials. Chem. Soc. Rev. 2015, 44, 5861-5896. 


\section{TABLE OF CONTENTS}

Graphene oxide crosslinked membranes stable in aqueous and non-aqueous solvents

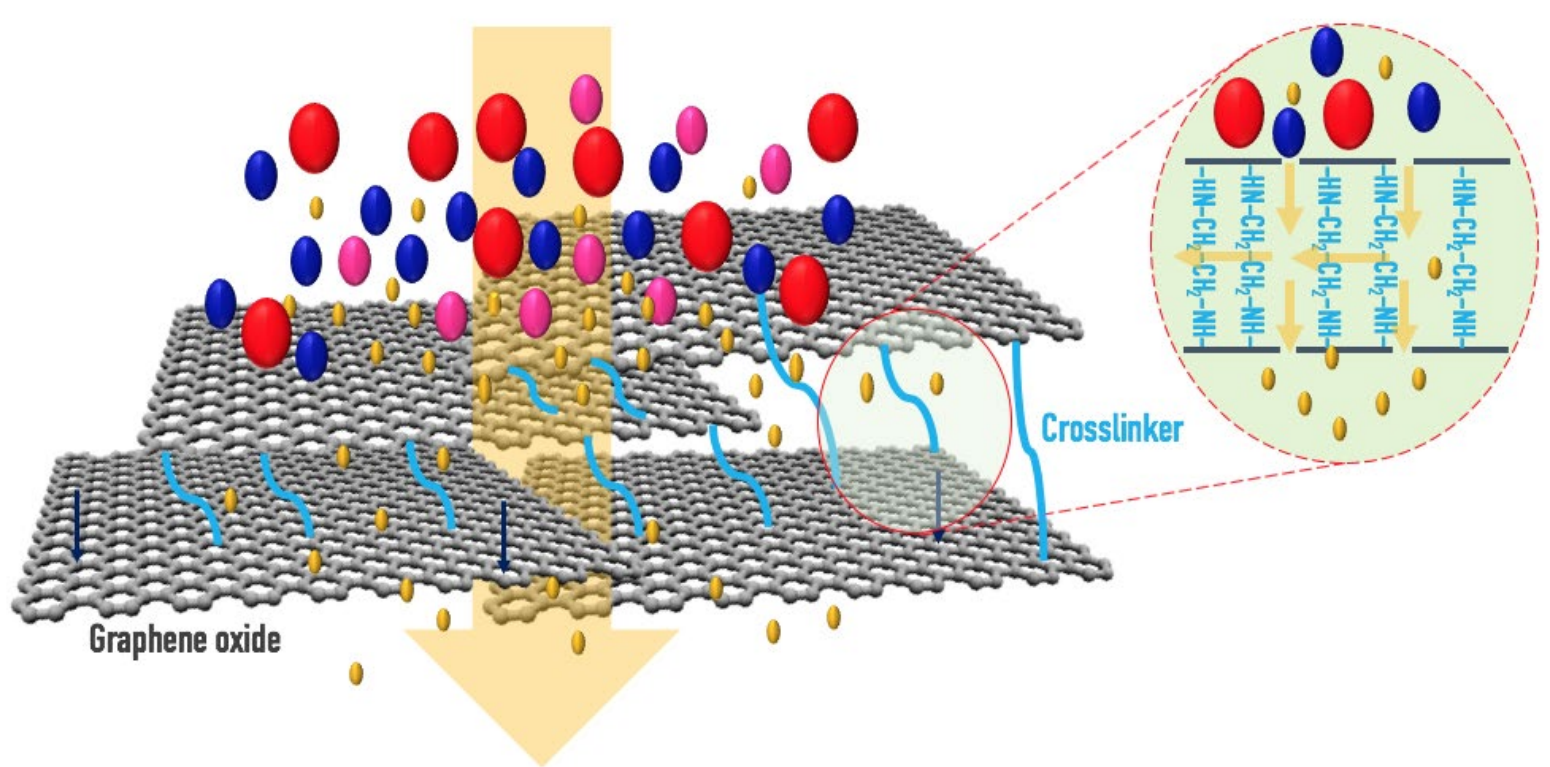

